Molecular Physics, 1985, Vol. 54, No. 1, 145-148

\title{
Approximate relation between the melting of hard spheres and ellipsoidal platelets and needles
}

\author{
by D. FRENKEL
}

Fysisch Laboratorium, Rijksuniversiteit Utrecht, P.O. Box 80000, 3508 TA Utrecht, The Netherlands

(Received 22 August 1984; accepted 7 September 1984)

\begin{abstract}
An approximate relation between the freezing point of a nematic fluid of extremely prolate (oblate) ellipsoids of revolution and the solid-liquid transition of hard spheres is presented. It is found that, in this approximation, flat and needle-like ellipsoids melt at the same packing fraction. The results are compared with recent Monte Carlo data.
\end{abstract}

From computer simulation studies much is known about the melting behaviour of model systems for simple liquids (for a review see e.g. [1]). In particular, due to the pioneering work of Hoover and Ree [2] the location of the melting point of hard spheres is known with high accuracy. In contrast, the study of the melting behaviour of systems consisting of non-spherical molecules is still at an early stage [3]. In this paper we present a simple approximate argument which relates the melting of hard spheres to that of hard ellipsoids of revolution with extreme ratio's between the length of the long and short axes.

An ellipsoid of revolution is a hard convex body. In a body-fixed frame its surface is described by the equation

$$
\left(x^{2}+y^{2}\right) / b^{2}+z^{2} / a^{2}=1 \text {. }
$$

In what follows we will concentrate on the properties of 'platelets' $(b / a \rightarrow \infty)$ and needles' $(a / b \rightarrow \infty)$. It is convenient to denote the length of the long axis ( $b$ for platelets, $a$ for needles) by $L$ and the short axis by $d$. The first point to note is that both needles and platelets undergo a transition to the nematic phase at a very Ww reduced density $\rho / \rho_{0}=O(d / L)[4,5]$; here $\rho_{0}$ is the density of the closeacked solid. In contrast, freezing takes place at a reduced density of order 1 . As consequence the ellipsoidal fluid is almost completely orientationally ordered at reezing. Here, and in what follows, we use the term 'ellipsoidal' to indicate both ery thin oblate and very long prolate ellipsoids of revolution, unless explicitly tated otherwise. Let us first consider the idealized situation that all molecules are erfectly aligned. In that case a simple change of scale, i.e. a compression of the peedles or an expansion of the platelets by a factor $L / d$ along the direction of lignment, with a corresponding transformation of all translational coordinates, reduces the system of hard ellipsoids to a system of hard spheres at the same acking fraction. Hence, apart from irrelevant constants, the translational part of The partition function of perfectly aligned ellipsoids is the same as for hard pheres [6]. Consequently, the solid-liquid transition of the aligned ellipsoid 
system occurs at the same reduced density and pressure as the melting of hard spheres. In practice the ellipsoidal system is only perfectly aligned at close packing. As the density decreases from close packing the alignment decreases and, as the density drops discontinuously at melting, so does the alignment. Clearly, there must be a discontinuous change in the orientational entropy at melting.

In order to proceed, we need an explicit expression for the density dependence of the orientational entropy. Below we derive a simple, approximate expression for this quantity. We start by considering the configurational part of the partition function of the dense, ellipsoidal fluid, $Q_{\mathrm{E}}\left(\rho / \rho_{0}\right) . Q_{\mathrm{E}}\left(\rho / \rho_{0}\right)$ is defined as

$$
Q_{\mathrm{E}}\left(\rho / \rho_{0}\right)=c \int d \mathbf{r}^{N} d \mathbf{\Omega}^{N} \exp \left(-\beta V\left(\mathbf{r}^{N}, \mathbf{\Omega}^{N}\right)\right)
$$

where $V$ is the potential energy of the $N$-particle system, $\mathbf{r}^{N}$ stands for all translational coordinates and $\boldsymbol{\Omega}^{N}$ for all orientational coordinates; $c$ is a numerical constant. Without loss of generality we can write

$$
Q_{\mathrm{E}}\left(\rho / \rho_{0}\right)=c Q_{\mathrm{Hs}}\left(\rho / \rho_{0}\right)\left\{Q_{\mathrm{E}}\left(\rho / \rho_{\mathrm{o}}\right) / Q_{\mathrm{Hs}}\left(\rho / \rho_{0}\right)\right\},
$$

where $Q_{\mathrm{HS}}\left(\rho / \rho_{0}\right)$ is the partition function of hard spheres at the same packing fraction. We now make the assumption that the aligned ellipsoidal system can serve as reference system for the real ellipsoidal fluid [6]. This requires that the overwhelming majority of acceptable configurations of the ellipsoidal system can be prepared by first distorting a hard-sphere system along a given axis and then rotating the individual ellipsoids over small angles $\theta_{i}$ with respect to this average alignment axis. It should be stressed that there exist configurations which cannot be prepared in this manner, i.e. states which, if the reverse process were applied, would result in a forbidden (overlapping) hard-sphere configuration. For the moment, however, we assume that the translational coordinates of the ellipsoidal system occupy a subspace of the translational phase-space of the (scaled) hardsphere system. In that case the quantity $Q_{\mathrm{E}}\left(\rho / \rho_{0}\right) / Q_{\mathrm{HS}}\left(\rho / \rho_{0}\right)$ is equal to

$$
Q_{\mathrm{R}}\left(\rho / \rho_{0}\right) \equiv \int P_{0}\left(\boldsymbol{\Omega}^{N}\right) d \mathbf{\Omega}^{N}
$$

In equation (4) $P_{0}\left(\boldsymbol{\Omega}^{N}\right)$ is the probability of finding a given set of molecular orientations $\left(\boldsymbol{\Omega}^{N}\right)$, free of overlap. The translational coordinates have been averaged over all acceptable hard-sphere configurations. Next, we make the assumption that $P_{0}\left(\boldsymbol{\Omega}^{N}\right)$ may be factorized into 1-particle probability distribution functions $p\left(\boldsymbol{\Omega}_{i}\right)$

$$
P_{0}\left(\boldsymbol{\Omega}^{N}\right)=\prod_{i=1}^{N} p\left(\boldsymbol{\Omega}_{i}\right) .
$$

Finally, we need an expression for $p\left(\boldsymbol{\Omega}_{i}\right)$ in terms of properties of the hard-sphere reference system. We observe that rotating an aligned ellipsoid over a small angle $\theta$ increases its excluded volume by $\Delta v$, with $\Delta v \simeq(L / d) \theta v_{0}$, where $v_{0}$ is the proper volume of the ellipsoid. In the hard-sphere reference system the rotation of an aligned ellipsoid corresponds to the transformation of a sphere into a (biaxial) ellipsoid. We now approximate the probability $p\left(\boldsymbol{\Omega}_{i}\right)$ by the probability that this biaxial ellipsoid intersects none of the spheres surrounding it. The 
probability that no other sphere is found in a small volume $\Delta v$ around a given sphere is approximately equal to $\exp (-\rho g(\sigma) \Delta v)$, where $g(\sigma)$ is the value of the pair-distribution function at contact. Hence

$$
p\left(\boldsymbol{\Omega}_{i}\right) d \boldsymbol{\Omega}_{i} \simeq p(\theta) \sin \theta d \theta d \phi=\exp \left(-\rho / \rho_{0} g(\sigma)(L / d) \theta\right) \sin \theta d \theta d \phi .
$$

With this approximation for $p\left(\boldsymbol{\Omega}_{i}\right)$ we obtain the following expression for $Q_{\mathbf{E}}\left(\rho / \rho_{0}\right)$

$$
Q_{\mathrm{E}}\left(\rho / \rho_{0}\right)=c Q_{\mathrm{HS}}\left(\rho / \rho_{0}\right) \cdot\left(4 \pi \int_{0}^{\infty} \exp \left(-\rho / \rho_{0} g(\sigma)(L / d) \theta\right) \theta d \theta\right)^{N},
$$

where we have made use of the fact that $\theta$ is small $(O(d / L))$. The expression for the free energy of the ellipsoidal system then becomes

$$
F_{\mathrm{E}}=a+F_{\mathrm{HS}}\left(\rho / \rho_{\mathrm{O}}\right)+2 N k T \ln \left(\rho / \rho_{\mathrm{O}} g(\sigma)\right) .
$$

In equation (8) all irrelevant additive constants have been lumped into $a$.

From equation (8) we can compute the pressure $(P=-(\partial F / \partial V))$ and the chemical potential (to within an additive constant). Using the MD data of [7] for $F_{\text {HS }}$ and $\rho g(\sigma)$ for both the hard-sphere solid and liquid, we can now compute the solid liquid transition in the ellipsoidal system. The results are given in the table. As the above derivation did not distinguish between needles and platelets, the data in the table apply to both cases. This means that we should expect the melting transition for needles and platelets to take place at the same packing fraction and reduced pressure. In the figure we compare the present predictions for extremely anisometric hard ellipsoids with recent Monte Carlo data on the melting transition of hard ellipsoids of revolution with finite $a / b$-ratio's [3]. In the Monte Carlo data we observe two trends which are also reproduced by the present approximate calculation: (1) there is a high degree of symmetry in the phase diagram under permutation of $a / b$ and $b / a$ and (2) the density of the melting transition appears to decrease as the molecules become more anisometric, at least for $a / b>2$ and $a / b<0.5$.

The present argument to predict the melting point of platelets and needles is simple but not rigorous. One consequence is that we predict a reduced density of the solid phase at coexistence which, at least for hard spheres, is at the limit of mechanical stability. It seems likely that a more systematic perturbative approach will result in somewhat different numerical values for the freezing densities and pressures of needles and platelets, but we expect that the qualitative aspects of the present description will prove to be correct.

Estimated coexistence properties of extremely oblate and prolate ellipsoids $(L / d=\infty) \mathrm{com}$ pared with the known hard-sphere $(L / d=1)$ coexistence properties [2]. $\rho_{\mathrm{S}}=$ density of solid phase at coexistence. $\rho_{\mathrm{L}}=$ density of fluid phase at coexistence. $P=$ pressure at solid-liquid coexistence. $\rho_{0}=$ density at close packing.

\begin{tabular}{lc:c}
\hline & $(L / d)=1$ & $(L / d)=\infty$ \\
\hline$\rho_{\mathrm{S}} / \rho_{0}$ & 0.736 & 0.670 \\
$\rho_{L} / \rho_{0}$ & 0.667 & 0.584 \\
$P / \rho_{0} k T$ & 8.27 & 8.41 \\
\hline
\end{tabular}




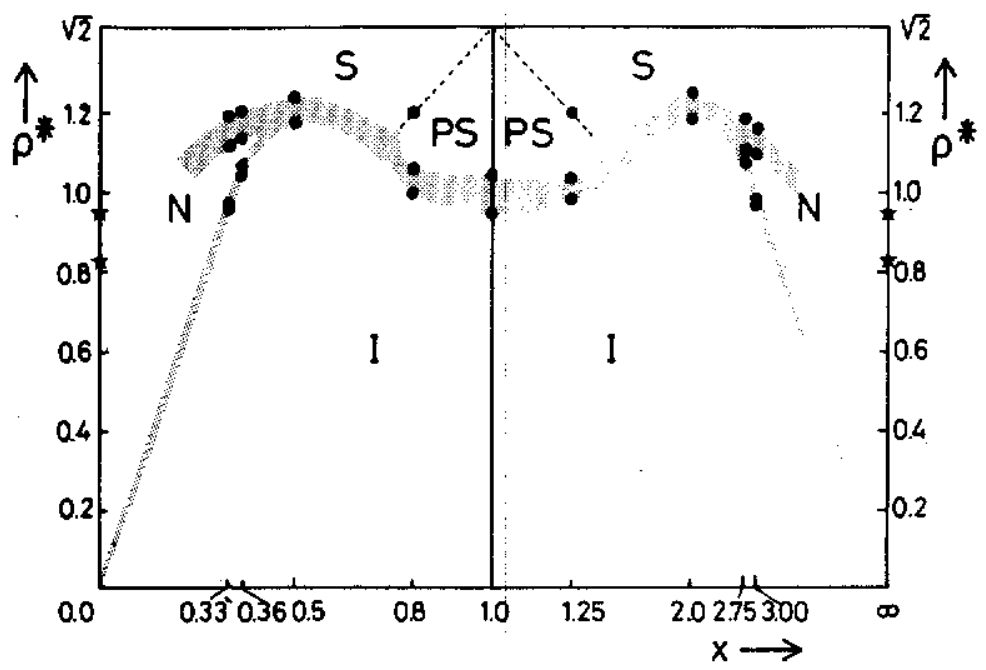

Phase diagram of hard ellipsoids of revolution. The axial ratio $a / b$ is denoted by $x$. The reduced density $\rho^{*}=(N / V) .\left(8 a b^{2}\right)$. Shaded areas are coexistence regions. The filled circles are Monte Carlo points from [3], except for the $a / b=1$ data (hard spheres), which were taken from [2]. The present estimates for the densities at coexistence of platelets and needles are indicated by stars. Four distinct phase are indicated in the phase diagram: $I=$ isotropic fluid, $N=$ nematic fluid, $S=$ ordered solid and $P S=$ plastic solid.

\section{REFERENCES}

[1] Frenkel, D., and McTague, J. P., 1980, A. Rev. phys. Chem., 31, 491.

[2] Hoover, W. G., and ReE, F. H., 1968, J. chem. Phys., 49, 3609.

[3] Frenkel, D., Mulder, B. M., and McTague, J. P., 1984, Phys. Rev. Lett., 52, 287. Frenkel, D., Mulder, B. M., and McTAgue, J. P., Molec. Crystals liq. Crystals (in the press).

[4]. Onsager, L., 1949, Ann. N.Y. Acad. Sci., 51, 627.

[5] Eppenga, R., and Frenkel, D., 1984, Molec. Phys., 52, 1303.

[6] Lebowitz, J. L., and Perram, J. W., 1983, Molec. Phys., 50, 1207.

[7] Alder, B. J., Hoover, W. G., and Young, D. A., 1968, 7. chem. Phys., 49, 3688. 